\title{
Possibility of Stars Energy Mechanism on the Earth
}

\author{
S. Davood Sadatian* \\ Department of Physics, Faculty of Basic Sciences, University of Neyshabur, \\ P. O. Box 91136-899, Neyshabur, Iran \\ *E-mail address: sd-sadatian@um.ac.ir
}

\begin{abstract}
Since long time ago, human's ambition has been obtaining an energy source which can be used for a long time, additionally it does not produce dangerous wastes. Todays in third millennium AD, it gradually seems to realize this unattainable ambition. Now human is getting ready for realizing his ancestors' dreams by making the first star stracture of energy. A pure and cheap fuel, named Hydrogen and rich production energy and a pure waste, named Helium. However, in this essay we explain some possibility of fusion method from stars on the earth.
\end{abstract}

Keywords: Fusion; Energy; star Formation

\section{INTRODUCTION}

\subsection{Fusion Method}

To make the fusion possible, the nuclei, which enter the reaction, must have sufficient kinetic energy to overcome peripheral electrostatic field. Therefore the temperatures associated to fusion reactions are so high[1].

\section{The method of utilizing energy in powerhouses}

(1) Nuclear Fusion: By colliding two types of Hydrogen (Deuterium \& Tritium), a Helium atom and an additional particle, Neutron is formed. In this transformation, a large amount of energy is released in form of light and heat. This process is called Fusion.

\section{The advantage of fusion}

(1) The fuel sources of nuclear fusion are abundant. For example, Deuterium constitutes about $0.0153 \%$ atoms of Hydrogen in oceans water. Tritium can also be produced in Neutron absorption process by Lithium.

(2) For each nucleon of fuel material, production energy is more than other method.

(3) It does not have wastes problem.

(4) When probable events are occurred, generator is not out of control.

\section{Fusion methods}

There are two ways to get the essential pressure and temperature for fusion of Hydrogen[2]:

(1) Magnetic Enclosure: Magnetic and electronic fields are used to heat and compress Hydrogen plasma in France (ITER project).

(2) Inertia Enclosure: Laser ray or ionic ray is used for heating Hydrogen plasma. 


\section{Initially, we examine magnetic enclosure}

Microwaves, electrical radiations, and neutral particles of accelerators will heat Hydrogen gas flow. This heat transforms gas to plasma. Plasma is compressed by a strong magnetic field and its highconductivity. The most efficient magnetic field of this plasma is circular; Toric field in which ions move in spiral route. This device is called "Tokamak".

\section{Basic segments of Tokamak (ITER)}

(1) Vacuum Tube: it holds plasma and protects interaction enclosure.

(2) Neutral Beam Injector (cyclotron ion): particles injects beam from accelerator to plasma to help plasma to reach critical temperature.

(3) Spiral Magnetic Field: very strong magnetic behavior that limits form and content of applied plasma in magnetic field.

(4) Central Solenoid / Transformator: it supplies electricity for spiral magnetic field.

(5) Cooling System: it cools magnet.

(6) Insulated System: it is made of Lithium. It absorbs high energy and heat of Neutron from fusion device.

(7) Divertor: it removes Helium products from fusion device.

\section{We examine inertia enclosure method}

(1) Fusion method will heat fuel Deuterium and Tritium in form of high temperature plasma. Plasma is compressed and fusion is occurred. The essential force to initiate fusion reaction is about $70 \mathrm{MB}$. But this reaction output is about $500 \mathrm{MB}$. Fusion reaction will last about 300 to 500 seconds.

(2) External Lithium coating of plasma interaction enclosure will absorb high energy of Neutron from fusion reaction to produce more Tritium fuel. Moreover, Lithium coating is heated through Neutron.

(3) Heat is transferred by cooling loop of thermal water exchanger and is transformed to steam.

(4) Steam moves electrical turbines to produce electricity.

(5) Steam is condensed and transformed to water to absorb more heat in thermal exchanger.

Initially ITER Tokamak tests suitability of fusion device and finally is transformed to a test fusion powerhouse.

Types of exothermic reactions

(1) Fusion in the Sun.

(2) Controlled Bootstrapping.

\subsection{Fusion in Sun and stars}

For many years, the scientists have discovered a reaction which occurs in the sun and stars and the energy is produced. This reaction includes synthesis (collision) of 4 common Hydrogen nuclei and production of a Helium nucleus. But there is a problem in this theory. The highest temperature in the sun relates to its center which equals to $15 \times 10^{6}$. This temperature reaches to $20 \times 10^{6}$ in larger stars. Because of this, it is supposed that the known reaction of 4 Hydrogen atoms synthesis and a Helium atom production in other large stars does not cause energy production. But probably carbon cycle helps to sustain their activity. The intention of carbon cycle is not a cycle which occurs on the earth, but at first, a normal Hydrogen atom synthesizes to a $\mathrm{C} 12$ atom (fusion) and a N13 atom along with a gamma beam unit are released. Then this atom is transformed to $\mathrm{C} 13$ atom, in addition to a Positron and a Neutrino by decomposition. Then this C13 synthesizes to a Hydrogen atom once more, and 
N14 and a gamma unit is resulted. Again due to synthesis of this Nitrogen to normal Hydrogen, O15 atom and a gamma unit are released and C12 is decomposed and N15 in addition to a Positron and a Neutrino are created. Finally a normal Hydrogen C12 synthesizes to N15 and a Helium atom is obtained [3].

$$
\begin{aligned}
& \mathrm{C}^{12}+\mathrm{H}^{1}=\mathrm{N}^{13}+\text { gamma } \\
& \mathrm{N}^{13}=\mathrm{C}^{13}+\mathrm{e}^{+}+\text {Neutrino } \\
& \mathrm{C}^{13}+\mathrm{H}^{1}=\mathrm{N}^{14}+\text { gamma } \\
& \mathrm{N}^{14}+\mathrm{H}^{1}=\mathrm{O}^{15}+\text { gamma } \\
& \mathrm{O}^{15}=\mathrm{N}^{15}+\mathrm{e}^{+}+\text {Neutrino } \\
& \mathrm{N}^{15}+\mathrm{H}^{1}=\mathrm{C}^{12}+\mathrm{He}^{4}
\end{aligned}
$$

As you saw, in this cycle, C12 neither was consumed, nor was created. But it only played the role of catalyzer. These reactions are performed respectively and successively. And the fundamental reaction is transformation of 4 Hydrogen atoms to a Helium atom. The advantage of carbon cycle is that it accelerates the process so much. But the problem is that in the least temperature, $20 \times 10^{6}$ is initiated. So it is so likely that in larger stars, carbon cycle causes energy production.

\section{Thermo reactions in internal zones of stars have dual effect of nucleus}

- Heavier atoms of initial Hydrogen are produced and nuclei are formed (nucleosynthesis).

- Hybrid energy is released and is transformed to those radiations.

- Stars lifetime and the existence of all chemical elements but light elements are the result of strong interactions between nucleons in interior depths of stars.

- The structure and evolution of stars and also formation of heavier elements relate to thermo reactions.

The essential temperature to initiate fusion must be about the temperature of the sun center, 15000000 kelvin or more.

\section{Proton-Proton Continuum}

The sun is a huge device in which existent Hydrogen nuclei are fused to form Helium nuclei. This thermo Hydrogen fuel goes through 3-step process which is called Proton-Proton Continuum.

\section{Different steps of Proton-Proton continuum}

Step 1: Step 1 includes 2 Protons fusion which terminates at forming a Deuterium and removing an anti-electron and a Neutron simultaneously. Almost immediately anti-electron collides with one of electrons in plasma and is destroyed by that electron and leads to two gamma beam emission. $\mathrm{H}+1 \mathrm{H}=2 \mathrm{H}+\mathrm{e}+\mathrm{y}(1.19 \mathrm{mev} 1)$

Step 2: The next step includes fusing hydrogen with Deuterium and producing Helium 


\section{3. $\mathrm{H}+2 \mathrm{H}=3 \mathrm{H}+\mathrm{e}+\mathrm{y}(1.19 \mathrm{mev} 1)$}

Step 3: It includes fusing two Helium 3 nuclei which terminates at common Helium formation (Helium 4) and removing two high energy Protons simultaneously. Since 2 classes of Helium 3 are needed in final stage, it is necessary that previous stages would have occurred twice, so final stage can be occurred once. Hence Proton-Proton continuum consumes 4 Protons to create a Helium 4 nucleus.

\section{The resultant Neutrino in Step 1}

In Step 1, Neutrino is released. Therefore, the center of the sun is not only the source of the heat, but also a flux of Neutrinos. Because intervention between Neutrinos and the material is so weak, the existent matter in the sun (and on the earth) for Neutrinos is almost transparent and these particles are flowed from the sun center to outside with no barrier.

\section{Carbon Cycle:}

Thermo burning of Hydrogen in the sun may be done via 6-step process which is called Carbon Cycle.

$$
\begin{gathered}
1 \mathrm{H}+12 \mathrm{C} 13 \mathrm{~N}+\mathrm{y}(1.95 \mathrm{MeV}) \\
13 \mathrm{~N}=13 \mathrm{C}+-\mathrm{e}+\mathrm{r}(2.22 \mathrm{Mev}) \\
1 \mathrm{H}+13 \mathrm{C}=14 \mathrm{~N}+\mathrm{y}(7.54 \mathrm{MeV}) \\
1 \mathrm{H}+14 \mathrm{~N}=15 \mathrm{O}+(7.35 \mathrm{MeV}) \\
15 \mathrm{O}=15 \mathrm{~N}+-\mathrm{e}+\mathrm{y}(2.71 \mathrm{MeV}) \\
11 \mathrm{H}+15 \mathrm{~N}=14 \mathrm{C}+6 \mathrm{He}(4.96 \mathrm{MeV})
\end{gathered}
$$

\section{Prevalent process in the Sun}

In the sun, the prevalent fusion process is the same Proton-Proton continuum, but in stars hotter that the sun, the prevalent process is Carbon Cycle. Variation of this prevalent process is because in normal temperatures higher (and faster) for Proton, it is prevailed and strong colony of 12 Carbons becomes simpler and this leads to perform the reaction in Step 1 of Carbon Cycle with a faster pace.

\subsection{Big Problem: Reaching to a high temperature on the earth}

Initiating fusion reaction requires so high temperature. It is true that 15 million degrees is so high temperature and the notion of creating it on the earth is difficult and also dreadful, but usually in daily routine life around us, there are extremely high temperatures which we neglect them. For example, when electricity wires in the box are burnt because of a contact and you hear its spark noise and after checking it, you realize that quite fused wires are just because of horrible temperature which occurred inside the box. This temperature reaches to about 30000-40000 degrees Kelvin[4]. Or we can pass electrical arcs through capillary pipes 
by using very high voltages. So air temperature inside the pipe which is transformed to plasma now, reaches to nearly multimillion (again it is low for fusion). One of the best ways is application of Laser. You know that lasers with extremely high powers have been made. For example, a type of laser named Nova Laser, can enhance energy equal to $10^{5} \mathrm{~J}$ in a while. But it also has drawbacks. For example, this laser naturally consumes so much energy; Even regardless of this problem, there is another problem. If production energy of laser in that short time must be delivered, so to sustain Lawson criterion (since enclosure time deducted), density must be increased; In this case is also higher than solid density and accumulation.

\section{RESULTS AND DISCUSSION}

\section{Reaction Types}

To optimize the performance of fusion devices and increase their output, there are various methods. One of them is selection of a reaction which is performed in reactor. The following reaction is a fusion reaction in which two light nuclei react together and create a heavier nucleus. That is, the result is synthesis of two Deuterium nuclei and production of a Tritium nucleus in addition to a normal Hydrogen nucleus. This energy reaction is ten. Because energy variation between heavier nucleus and lighter nuclei is negative. In this reaction, the amount of production energy equals to $4 \mathrm{MeV}$. It was mentioned that nuclei must sufficiently approach each other for fusion. This sufficient amount is about $3 \mathrm{fm}$. Because electrostatic potential energy of two Deuterons is about $0.5 \mathrm{MeV}$ in these intervals, so we can break colony repulsion between Neutrons through giving this amount of energy to one of Deuterons and initiating reaction, that since doing it, $4.5 \mathrm{Mev}$ is produced. $0.5 \mathrm{MeV}$ kinetic energy in addition to $4 \mathrm{MeV}$ released energy.

$$
{ }_{3}^{6} \mathrm{Li}_{3}+\mathrm{n} \longrightarrow{ }_{2}^{4} \mathrm{He}+{ }_{1}^{3} \mathrm{H}_{2}
$$

In Proton-Proton continuum, Protons are collided and fused to form complicated Helium atoms. In this process, they release high energy particles such as Neutrino, Positron and Feutron. We can design our own reactor so that liquid lithium under pressure is flowed around its external wall. This liquid Lithium absorbs additional production heat from reaction and transfers it to water and by transforming it to steam, it causes to move turbine and generator and to produce the electricity.

\section{Controlled bootstrapping}

NIF team's success is not in initiating reaction, but it is latent in its method, which is called fuel reinforcement. In this method, by using an array consist of 192 strong laser beams which are concentrated on a circle plastic piece containing a mixture of Deuterium and Tritium, the scientists have succeeded to supply a drop of freeze fuel which reaches to the sun temperature by applying $19 \mathrm{MJ}$ high energy in a fraction of a second. It resulted that finally energy out of system became more than applied energy to initiate reaction, that it has not been seen in any other laboratory. The other success was obtaining the energy 10 times more than other cases which had performed fusion reaction. The key of this adventure has also been the application of a method named controlled bootstrapping. Controlled bootstrapping is a meaningful term. In fusion devices, some system energy was transferred out of system by alpha particles (Helium particles without electron), which were high energy, that caused to decrease total energy of system and to re-enter energy from outside which directed energy 
equation in favor of consumption. In controlled bootstrapping, by using a mixture of Deuterium and Tritium atoms, these alpha particles were controlled that caused to increase plasma temperature and final increase of reaction. As these researchers said, the key point of performing controlled bootstrapping was keeping plastic crust containing fuel healthy to prevent its collapse during compressing stage by laser pulse. They believed that others were unsuccessful to perform fusion because of crust collapse which caused to stop reaction, and this problem was solved by controlling and changing the applied laser. The interesting point in this case is that this group observed self-heating of alpha particles by increasing reaction intensity via controlled bootstrapping. Of course, this group's main purpose was not obtaining energy [5].

\section{To energize}

Definitely an ideal fusion method is a reaction in which continuum reactions exist. In fact, the main purpose of creating a nuclear fusion device is making it chained. If we must consume more energy in this way to get less amount of it, definitely this reaction is neither a continuum, nor effective. The scientists in this field have defined a concept called catching (Giranesh), which means we should consume some amount of energy to initiate the reaction and get more energy from reactions series. In fact, in catching conditions, the reaction becomes chained. That is, not only production energy of a reaction is sufficient to perform the next reaction, but also a large amount of it is over plus and it can provide us electricity. If we want Tokamak or other devices in which fusion is performed, have sufficient output, that is, provide us energy, they must be in specific conditions. To high possible colliding between candidate particles (ions) of fusion, firstly, extremely high temperature must be produced inside it and the device can tolerate high temperature very well. (This temperature is about $10^{8}$ degree Kelvin). Secondly, the device must be able to admit high density of input ions, and thirdly, enclosure time inside it must be long. High temperature is to ensure passing through strong barrier of colonial potential of nuclei. High density is also for high probable efficient collisions. There is a rule in this route which is called Lawson Criterion. With this criterion, we can evaluate whether there are conditions in which the reaction reaches to catching or not. Lawson Criterion=it must: density amount*enclosure period time $>10^{20}$ particle in cubic meter, so that the reaction reaches to catching. Of course it directly depends on plasma temperature.

\section{Tokamak}

One of fusion processes was performed by a huge machine called Tokamak. Tokamak is a Russian term, meaning magnetic flux. The fundamental segment of this huge machine is a coiled passage, in which strong magnetic fields are established and plasma is heated over 300 million degrees within at least 2 seconds by motivating in these fields. This situation causes nuclear fusion of light atoms. The first practical result from this machine has exactly been the same as fusion reaction. The fusion reaction in this machine, which was performed by Russian physicists in the late 1950s, is progressed under control. In Tokamak, plasma is created due to electrical discharge in gas mixture, Deuterium-Tritium and is insulated inside magnetic and immaterial walls of magnetic fields against the heat. To explain the reason of the existence of these magnetic walls and indeed electromagnetic, the rules of electromagnetism theory must be considered, particularly a section related to the force over mobile charged particles in magnetic fields. To understand it well, we must remind you that a force importing on an electrical charge which enters into the field with perpendicular velocity on a magnetostatic field, that is both perpendicular on direction of magnetic field and on velocity direction of input charge into the field. A mechanical and mathematical analysis 
indicates that sustaining this charge movement in this field is in a circular route with a perpendicular surface over magnetic field.

\section{Fusion with a magnet}

Laser is not the only available method to perform fusion process. An international experimental thermo device (ITER) which is under production in France at present, applies a method in which Hydrogen fusion process is performed by a magnet. Former magnetic devices have just obtained thermal energy production and they could not have acted as an ignition system so far. Paul Thomas who was among the scientists of international reactor project said in response to publish the results of this study: "I appreciate NIF research team's efforts and encourage them to keep on acting in ignition field". Holicane added: Even if NIF group obtains to its own purpose and can control stars power, producing fuel and targeting laser beams is a slow and difficult process, and moreover, strong laser explosion decreases device speed so that it removes the process from business and causes to perform it in laboratories. Solving these kinds of problems is prerequisite to transform fusion energy to a practical solution to produce energy. This research group's article in Nature Magazine namely, "Fusion energy approaches 1 inch closer to be star" has been published.

\section{Spheromak, another type of fusion devices}

Another type of fusion devices is Spheromak, which unlike Tokamak, is circular. Of course, the difference between Tokamak and Spheromak is that there is no material and physical body in Spheromak center. Unfortunately, Spheromak was neglected and did not attract attentions as much as Tokamak. While Spheromak was invented a long time after Tokamak. In the last decade, the researchers in magnetic fusion energy field have often been focused on toricTokamak to reach a high level of fusion reactions. In SSPX project leader's opinion, Tokamak with a high temperature in which is available and obtainable (over 100 million ${ }^{0} \mathrm{c}$ which is more than temperature in sun center), is currently known as leadership of fusion projects. Anyway, magnetic fields of Tokamak are produced by large external coils which encompass reactor torque. These large coils will cause heavy costs and disorders and irregularity in device performance. While Spheromak creates so hot plasma in a simple and compressed magnetic field system which uses a simple series of small stable coils, the essential strong magnetic fields inside the plasma are produced by magnetic generator.

\section{CONCLUSIONS}

Fusion power methods are to be practical, it will still be necessary to convert the into usable energy form. However, it could be used for thermochemical processes such a transport fuels. In othe view, fusion has new problems because these influence the choice of materials and the operating temperature, determining ultimately the choice of coolant and the working cycle. The limited temperature ranges allowed by present day structural materials irradiated, combined with the large internal power demand of the plant, will limit the overall thermal efficiency. The operating conditions of the source of fusion energy, materials, liquid cooling, and energy conversion system will all need to be tightly integrated. 


\section{References}

[1] Taleyarkhan, R. P. et al. Science 295, 1868 (2002).

[2] K. Hlav'a`ckov'a-Schindler, M. Palu`s, M. Vejmelka, and J. Bhattacharya. Causality detection based on information-theoretic approaches in time series analysis, Phys. Reports, 441(1):1 (2007).

[3] T. Schreiber. Measuring information transfer. Phys. Rev. Lett., 85(2) 461 (2000).

[4] G. Kresse and J. Furthmuller, Comput. Mater. Sci.6 15 (1996).

[5] G. Kresse and J. Furthmuller, Phys. Rev. B 5411169 (1996). 